Jin-Ke Jiang, Xin-Yue Xu, Xi-Xi Li, Jing-Jie Du, Hui Jiang and Xian-Wu Dong*

\title{
Crystal structure of catena-poly[aqua $\left(\mu_{4}\right.$-piperazine-1,4-bis(2- hydroxypropanesulfonato- $\left.\mathrm{K}^{8} O, O^{\prime}: O^{\prime}, N: N^{\prime}, O^{\prime \prime}: O^{\prime \prime}, O^{\prime \prime \prime}\right)$ )silver(I)], $\mathrm{C}_{10} \mathrm{H}_{24} \mathrm{Ag}_{2} \mathrm{~N}_{2} \mathrm{O}_{10} \mathrm{~S}_{2}$
}

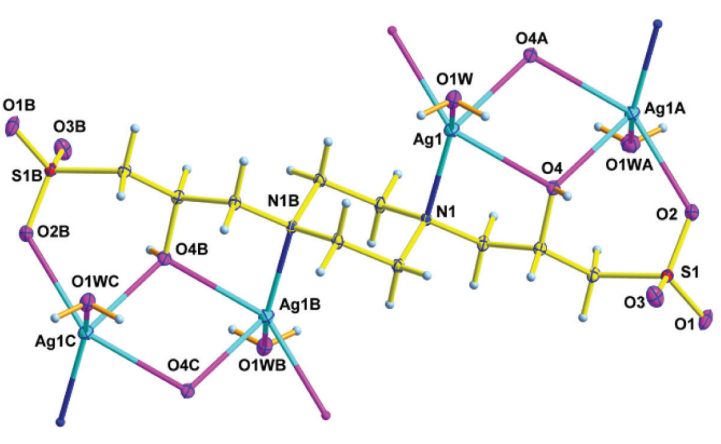

https://doi.org/10.1515/ncrs-2019-0610

Received August 22, 2019; accepted October 9, 2019; available online October 25, 2019

\section{Abstract}

$\mathrm{C}_{10} \mathrm{H}_{24} \mathrm{Ag}_{2} \mathrm{~N}_{2} \mathrm{O}_{10} \mathrm{~S}_{2}$, triclinic, $P \overline{1}$ (no. 2), $a=6.9547(9) \AA$, $b=8.4684(12) \AA, \quad c=8.9563(15) \AA, \quad \alpha=117.888(15)^{\circ}$, $\beta=101.740(13)^{\circ}, \quad \gamma=93.026(11)^{\circ}, \quad V=449.75(13) \AA^{3}, \quad Z=1$, $R_{\mathrm{gt}}(F)=0.0365, w R_{\text {ref }}\left(F^{2}\right)=0.0858, T=293(2) \mathrm{K}$.

\section{CCDC no.: 1946384}

Tables 1 and 2 contain details on crystal structure and measurement conditions and a list of the atoms including atomic coordinates and displacement parameters.

\section{Source of materials}

The $\mathrm{Ag}_{2} \mathrm{CO}_{3}$ (0.014 g, $0.05 \mathrm{mmol}$ ) and piperazine-1, 4-bis(2hydroxypropanesulfonic acid) $(0.040 \mathrm{~g}, 0.1 \mathrm{mmol})$ in $15 \mathrm{~mL}$

*Corresponding author: Xian-Wu Dong, College of Biology and Pharmaceutical Engineering, Jilin Agricultural Science and Technology University, Jilin 132101, P.R. China, e-mail: xianwu_jlnku@163.com. https://orcid.org/0000-00029492-8664

Jin-Ke Jiang, Xin-Yue Xu, Xi-Xi Li and Hui Jiang: College of Biology and Pharmaceutical Engineering, Jilin Agricultural Science and Technology University, Jilin 132101, Province Jilin, P.R. China. https://orcid.org/0000-0003-0156-3334 (H. Jiang)

Jing-Jie Du: College of Food Engineering, Jilin Agricultural Science and Technology University, Jilin 132101, Province Jilin, P.R. China

๑ Open Access. ( 2019 Jin-Ke Jiang et al., published by De Gruyter. (c) BY License.

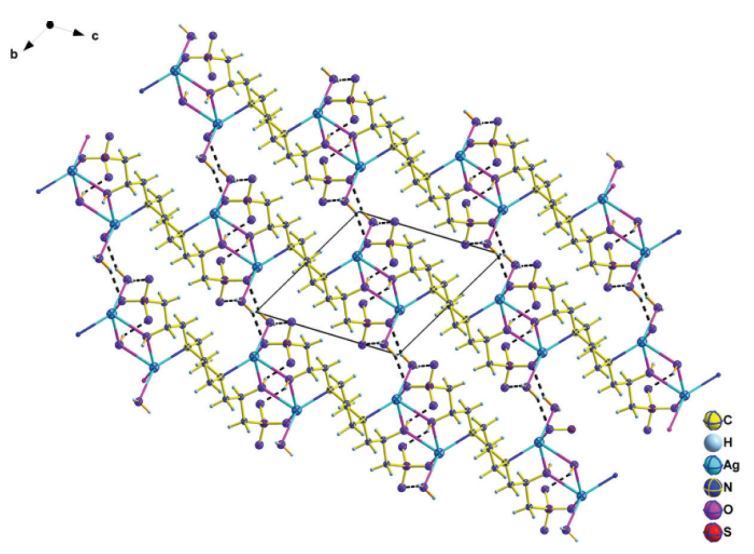

Table 1: Data collection and handling.

\begin{tabular}{|c|c|}
\hline Crystal: & Block, colorless \\
\hline Size: & $0.15 \times 0.12 \times 0.12 \mathrm{~mm}$ \\
\hline Wavelength: & Mo $K \alpha$ radiation $(0.71073 \AA$ ) \\
\hline$\mu:$ & $2.46 \mathrm{~mm}^{-1}$ \\
\hline Diffractometer, scan mode: & XtaLAB Pro, $\omega$-scans \\
\hline$\theta_{\max }$, completeness: & $29.8^{\circ}, 88 \%$ (up to $\left.25.0^{\circ},>99 \%\right)$ \\
\hline$N(h k l)_{\text {measured }}, N(h k l)_{\text {unique }}, R_{\text {int }}:$ & $6174,2258,0.055$ \\
\hline Criterion for $I_{\mathrm{obs}}, N\left(h k l_{\mathrm{gt}}\right.$ : & $I_{\text {obs }}>2 \sigma\left(I_{\text {obs }}\right), 1846$ \\
\hline$N(\text { param })_{\text {refined }}:$ & 123 \\
\hline Programs: & $\begin{array}{l}\text { CrysAlis }{ }^{P R O}[1], \text { SHELX }[2,4] \text {, } \\
\text { WinGX and ORTEP [3] }\end{array}$ \\
\hline
\end{tabular}

$\mathrm{H}_{2} \mathrm{O} / \mathrm{CH}_{3} \mathrm{CN}(2: 1, v / v)$ reacted for 25 minutes. The filtrate was kept at room temperature to produce colourless block crystals after several days.

\section{Experimental details}

Data were collected via CrysAlis ${ }^{\mathrm{PRO}} 1.171 .39 .7 \mathrm{e}$ [1], the structure of crystal was determined by SHELXT [2] within WinGX [3] and refined by SHELXL [4].

\section{Comment}

As illustrated in the left part of the figure, the structure of crystal compromises a unique $\mathrm{Ag}(\mathrm{I})$ center, one half of a piperazine-1, 4-bis(2-hydroxypropanesulfonato) ligand (pipS) and one water molecule. The Ag1 is five-coordinated by one nitrogen atom and three oxygen atoms from different pips 
Table 2: Fractional atomic coordinates and isotropic or equivalent isotropic displacement parameters $\left(\AA^{2}\right)$.

\begin{tabular}{lrrrr}
\hline Atom & $\boldsymbol{x}$ & $\boldsymbol{y}$ & $\boldsymbol{z}$ & $\boldsymbol{U}_{\text {iso }}{ }^{*} \boldsymbol{U}_{\text {eq }}$ \\
\hline Ag1 & $-0.00140(5)$ & $0.65550(4)$ & $0.76175(4)$ & $0.04218(12)$ \\
N1 & $0.0416(4)$ & $0.4230(4)$ & $0.8290(3)$ & $0.0231(5)$ \\
O1 & $0.4987(5)$ & $-0.0031(4)$ & $0.2480(4)$ & $0.0476(8)$ \\
O2 & $0.3240(4)$ & $0.2243(4)$ & $0.2225(4)$ & $0.0409(7)$ \\
O1W & $0.1604(4)$ & $0.9530(4)$ & $0.8671(4)$ & $0.0396(6)$ \\
H1A & 0.270933 & 0.952296 & 0.834559 & $0.059^{*}$ \\
H1B & 0.201681 & 1.009151 & 0.981200 & $0.059^{*}$ \\
O3 & $0.6039(4)$ & $0.3107(4)$ & $0.4697(4)$ & $0.0443(7)$ \\
O4 & $0.1815(4)$ & $0.4387(3)$ & $0.5520(3)$ & $0.0317(5)$ \\
S1 & $0.43913(13)$ & $0.17156(11)$ & $0.34130(11)$ & $0.02623(18)$ \\
H10 & $0.297(12)$ & $0.513(12)$ & $0.579(11)$ & $0.12(3)^{*}$ \\
C1 & $0.2790(5)$ & $0.1422(5)$ & $0.4605(4)$ & $0.0269(7)$ \\
H1 & 0.149678 & 0.077698 & 0.380436 & $0.032^{*}$ \\
H2 & 0.332499 & 0.066785 & 0.507980 & $0.032^{*}$ \\
C2 & $0.2504(5)$ & $0.3184(4)$ & $0.6103(4)$ & $0.0239(6)$ \\
H3 & 0.375306 & 0.376693 & 0.702347 & $0.029^{*}$ \\
C3 & $0.0892(5)$ & $0.2688(4)$ & $0.6814(4)$ & $0.0259(7)$ \\
H4 & -0.031559 & 0.208768 & 0.587169 & $0.031^{*}$ \\
H5 & 0.131646 & 0.182514 & 0.718458 & $0.031^{*}$ \\
C4 & $0.2008(5)$ & $0.4916(5)$ & $0.9915(4)$ & $0.0272(7)$ \\
H6 & 0.322808 & 0.536993 & 0.977145 & $0.033^{*}$ \\
H7 & 0.225409 & 0.393490 & 1.016294 & $0.033^{*}$ \\
C5 & $0.1419(5)$ & $0.6418(5)$ & $1.1424(4)$ & $0.0273(7)$ \\
H8 & 0.249497 & 0.687387 & 1.247776 & $0.033^{*}$ \\
H9 & 0.120434 & 0.740797 & 1.118380 & $0.033^{*}$ \\
\hline
\end{tabular}

ligands including two oxygen atoms from the same ligand and one oxygen atom from water to construct a chain structure [5]. At the same time, the structure contains $\mathrm{OH} \cdots \mathrm{O}$ interactions connecting adjacent chains [6] and the $\mathrm{Ag}_{2} \mathrm{O}_{2}$ moiety is well known [7] ( $c f$. right part of the figure). (symmetry codes: $\mathrm{A}=-x, 1-y, 1-z ; \mathrm{B}=-x, 1-y, 2-z ; \mathrm{C}=x, y, 1+z)$.

Acknowledgements: This work was supported by the Jilin Agricultural Science and Technology University Students Science and Technology Innovation and Entrepreneurship Training Program Project (no. 2019034).

\section{References}

1. Rigaku Corporation. CrysAlis ${ }^{\mathrm{PRO}}$. Yarnton, Oxfordshire, England (2015).

2. Sheldrick, G. M.: SHELXT - Integrated space-group and crystalstructure determination. Acta Crystallogr. A71 (2015) 3-8.

3. Farrugia, L. J.: WinGX and ORTEP for Windows: an update. J. Appl. Cryst. 45 (2012) 849-854.

4. Sheldrick, G. M.: Crystal structure refnement with SHELXL. Acta Crystallogr. C71 (2015) 3-8.

5. Wu, H.; Dong, X.-W.; Liu, H.-Y.; Ma, J.-F.; Liu, Y.-Y.; Liu, Y.-Y.; Yang, J.: Construction of a series of inorganic-organic hybrid coordination polymers based on hexamethylenetetramine and sulfonate ligands. Inorg. Chim. Acta 373 (2011) 19-26.

6. Wu, H.; Lu, X.-L.; Lu, B.; Dong, C.-X.; Wu, M.-S.: A novel threedimensional $\mathrm{Ag}^{\prime}$ coordination polymer based on mixed naphthalene-1,5-disulfonate and aminoacetate ligands. Acta Crystallogr. C69 (2013) 876-879.

7. Fan, H.-T.; Ding, J.; Xie, J.-P.; Wang, K.: Crystal structure of bis\{catena poly $\left[\left(\mu_{2}-1,2-\right.\right.$ bis (4-pyridyl)ethane- $\left.\left.\left.{ }^{2} N: N^{\prime}\right) \operatorname{silver}(I)\right]\right\}$ diaqua -bis(5-(4-carboxyphenyl)pyridine-2-carboxylato- $\left.\mathrm{K}^{2} \mathrm{~N}, \mathrm{O}\right)$ $\left(\mu_{2}\right.$-1,2-bis(4-pyridyl)ethane- $\left.{ }^{2} N: N^{\prime}\right)$ disilver $(I)$ octahydrate, $\mathrm{C}_{31} \mathrm{H}_{35} \mathrm{Ag}_{2} \mathrm{~N}_{4} \mathrm{O}_{9}$. Z. Kristallogr. NCS 233 (2018) 585-587. 